\title{
Une routine de la civilité épistolaire : l'expression de la condoléance
}

\section{Cécile Lignereux}

\section{(2) OpenEdition}

1 Journals

Édition électronique

URL : http://journals.openedition.org/rhetorique/437

DOI : $10.4000 /$ rhetorique.437

ISSN : 2270-6909

Éditeur

UGA Éditions/Université Grenoble Alpes

Édition imprimée

ISBN : 978-2-84310-316-2

Référence électronique

Cécile Lignereux, «Une routine de la civilité épistolaire : l'expression de la condoléance », Exercices de rhétorique [En ligne], 6 | 2016, mis en ligne le 08 février 2016, consulté le 12 septembre 2020. URL http://journals.openedition.org/rhetorique/437 ; DOI : https://doi.org/10.4000/rhetorique.437

Ce document a été généré automatiquement le 12 septembre 2020.

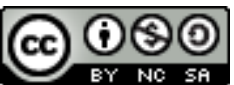

Les contenus de la revue Exercices de rhétorique sont mis à disposition selon les termes de la Licence Creative Commons Attribution - Pas d'Utilisation Commerciale - Partage dans les Mêmes Conditions 4.0 International. 


\title{
Une routine de la civilité épistolaire : l'expression de la condoléance
}

\author{
Cécile Lignereux
}

1 Le 21 octobre 1671, Mme de Sévigné écrit à Mme de Grignan, qui attend alors son deuxième enfant :

Ma bonne, que votre ventre me pèse! et que vous n'êtes pas seule qu'il fait étouffer ! (21 octobre $1671:$ I, 366 ${ }^{1}$ )

Fondés eux aussi sur de curieux mécanismes de transfert entre la première et la deuxième personne, d'autres passages de la correspondance semblent confirmer la capacité de Mme de Sévigné à se mettre à la place de sa fille, au point de partager ses sensations - que celles-ci soient liées à la pluie qui accroît l'inconfort d'un voyage,

Pour vous, ma chère bonne, je vous crois partie. Il ne tombe pas une goutte de pluie qui ne me fasse mal. (26 janvier 1674 : I, 684)

à la violence du vent de Provence,

La bise de Grignan [...] me fait mal à votre poitrine [...]. (29 décembre $1688:$ III, 450)

ou à l'inconfort du palais qu'habite la comtesse lorsqu'elle réside à Aix :

Faites donc de l'exercice, car c'est mourir que d'être toujours dans ce trou de

cabinet ; j'en étouffe. (11 février 1689 : I, 502)

Face à l'expression de tant de sympathie ${ }^{2}$ (au sens étymologique de participation à la souffrance d'autrui), grande est la tentation de céder au commentaire psychologique, à l'instar de l'un des lecteurs de la Correspondance les plus fervents du XIX ${ }^{\mathrm{e}}$ siècle, Alphonse de Lamartine, célébrant «cette connexité absolue d'existence et cet anéantissement complet de sa personnalité dans une autre ${ }^{3}$ ». S'ils font preuve d'un lyrisme plus mesuré, les critiques du $\mathrm{XX}^{\mathrm{e}}$ siècle ne rompent pas pour autant avec un mode de lecture psycho-biographique prompt à considérer les sentiments qui s'expriment dans les lettres à Mme de Grignan comme autant d'affects incontrôlables, symptomatiques d'un état passionnel. C'est ainsi qu'en 1988, Michèle Longino analyse l'entreprise épistolaire de Mme de Sévigné comme un phénomène de vie par substitution fondée sur un phénomène de "vicariance ${ }^{4}$ "; qu'en 1996, Patrick Avrane diagnostique un cas de «transitivisme» - terme qui, en psychanalyse, désigne le 
«passage, sans intermédiaire, des émotions de l'un à l'autre ${ }^{5}$ »; qu'en 1997, MariePierre Pasquini-Butts démontre que la passion de Mme de Sévigné pour sa fille donne lieu à une forme de "cannibalisme ", dans la mesure où l'épistolière "incorpore " sa fille à son texte de manière à la "neutraliser ${ }^{6}$ "; qu'en 2009 enfin, Nathalie Freidel, commentant les «phénomènes identificatoires» résultant de la «faculté de sympathiser avec sa correspondante ", remarque que la manière dont "l'épistolière ressen[t] réellement les souffrances de son interlocutrice, n'est pas sans rappeler ces expériences limites dans lesquelles le mystique découvre sur son propre corps les stigmates de la crucifixion ${ }^{7} »$. Aussi diverses que soient ces approches, toutes présentent l'inconvénient de réduire l'expression de la compassion pour les maux de Mme de Grignan à des traces d'affects paroxystiques - les analyses mentionnées interprétant volontiers la sympathie maternelle, à grands renforts de grilles psychanalytiques, en termes de fusion passionnelle, de contagion émotionnelle ou de symbiose mère-fille.

2 C'est oublier un peu vite que les sentiments qui s'expriment dans les lettres non seulement subissent nécessairement l'influence des modèles axiologiques qui président à une nomenclature des émotions socio-culturellement déterminée, mais encore que dans le cadre d'une correspondance, les mouvements de sensibilité ne sauraient être communiqués en-dehors de rouages discursifs scrupuleusement normalisés. Dans l'appréciation des témoignages de tendresse qui scandent les lettres à Mme de Grignan, l'ignorance du contexte anthropologique propre à la politesse mondaine en général et aux pratiques de civilité épistolaire en particulier est à l'origine de bien des contresens. À rebours des études qui appréhendent les formules de compassion comme l'une des manifestations irrépressibles d'un amour maternel d'une intensité exceptionnelle, il s'agit donc de procéder à leur réévaluation - et pour cela d'identifier les praxis comportementales et langagières qu'elles mobilisent. C'est pourquoi nous recenserons les préconstruits socio-discursifs sous-jacents à l'expression de la sympathie avant d'étudier les marqueurs stylistiques des séquences de condoléance dans les lettres à Mme de Grignan.

\section{Les préconstruits socio-discursifs sous-jacents à l'expression de la sympathie}

3 Restituer aux formules de compassion leur juste fonctionnalité au sein des lettres familières adressées à Mme de Grignan implique d'une part, de les rapporter aux modèles de comportement qui façonnent les relations intersubjectives ; d'autre part, de les replacer dans le contexte des pratiques réglées de la bienséance épistolaire ; enfin, de les confronter aux représentations topiques propres au discours de condoléance.

\subsection{Les modèles de comportement valorisant la participation aux maux d'autrui}

Plutôt que de considérer la compassion dont fait preuve Mme de Sévigné comme un état émotionnel spontanément éprouvé par le sujet écrivant, il convient de souligner la dialectique qui s'exerce entre d'un côté, une expérience subjective (l'angoisse d'une mère, encore accrue par la distance, causée par les différents périls qui menacent objectivement sa fille, au premier rang desquels ses problèmes de santé et l'état 
désastreux de ses finances) et de l'autre, des normes psycho-affectives qui valorisent la participation aux souffrances d'autrui - celles de l'amitié d'une part et celle de la tendresse d'autre part.

Premièrement, en faisant preuve de sympathie à l'égard des maux dont souffre Mme de Grignan, l'épistolière incarne l'une des attitudes constitutives de l'amitié, à une époque où celle-ci est conçue comme un mode de relation reposant sur des usages étroitement normalisés. Loin de se réduire à un rapport sentimental fondé sur le seul ressenti intime, l'amitié est indissociable des signes tangibles qui la prouvent et la concrétisent. Parmi ce que les ouvrages de savoir-vivre, de morale et d'art épistolaire désignent comme des marques d'amitié - qui comprennent aussi bien des qualités morales que des attitudes comportementales et des pratiques langagières - figurent précisément le partage et la continuité intime des pensées, conçus en termes de communication ${ }^{8}$, étant admis à titre de principe qu' « il faut communiquer à nos amis, nos joyes, nos afflictions, nos plus secrettes affaires ${ }^{9}$ ». À l'opposé d'une réaction émotionnelle intempestive, la sympathie qui résulte d'un tel partage ${ }^{10}$ apparaît comme une composante définitoire de l'amitié, qui doit être soigneusement cultivée - les traités sur l'amitié insistant volontiers sur le fait que «le premier fruit que les amis doivent tirer de la communication de leurs pensées, c'est une vive attention sur tout ce qui les interesse " et qu' "être inquiet [des] moindres maux" d'un ami, "c'est sçavoir repandre les douceurs de l'amitié sur toutes les parties de la vie ${ }^{11} »$.

6 Deuxièmement, en témoignant régulièrement sa compassion à l'égard de Mme de Grignan, Mme de Sévigné exhibe les preuves d'une tendresse authentique, conforme à la célèbre définition qu'en donne Mlle de Scudéry, qui non seulement caractérise les personnes incapables d'être tendres par le fait que " les médiocres maux de ceux qu'ils aiment ne les touchent guère ", mais encore fait de la sympathie l'un des critères de la véritable tendresse, en affirmant que seules les âmes tendres «sentent si vivement toutes les douleurs, et toutes les joies de ceux qu'elles aiment, qu'elles ne sentent pas tant les leurs propres ${ }^{12} »$.

7 Lorsque Mme de Sévigné déclare par exemple «suivre» sa fille «dans [ses] jours d'inquiétude ",

Je vous ai suivie, ma bonne, dans tous vos jours d'inquiétude. (6 octobre 1680 : III,

elle ne fait pas qu'avouer les angoisses qui la rongent. Elle « active à travers la lecture » un «monde éthique », défini comme " un stéréotype culturel qui subsume un certain nombre de situations stéréotypiques associées à des comportements ${ }^{13}$ ». Ainsi envisagée à l'aune des modèles éminemment valorisés de l'amitié et de la tendresse, la compassion qui s'exprime dans les lettres à Mme de Grignan s'avère dotée d'une incontestable dignité morale. D'ailleurs, Mme de Sévigné ne manque pas de souligner combien les mouvements de sympathie que provoquent en elle les maux de sa fille constituent d'indubitables preuves de la qualité de ses sentiments ${ }^{14}$ :

Quoi ! ma bonne, vous avez pensé brûler, et vous voulez que je ne m'en effraye pas!

Vous voulez accoucher à Grignan, et vous voulez que je ne m'en inquiète pas ! Ma

bonne, priez-moi, en même temps, que je ne vous aime guère. Mais soyez assurée que pendant que vous me serez ce que vous êtes à mon cœur, c'est-à-dire pendant que je vivrai, je ne puis jamais voir tranquillement tous les maux qui vous peuvent arriver. (16 août $1671: \mathrm{I}, 321$ )

Il faut que j'entre dans vos peines. L'amitié le veut ainsi. ( $1^{\text {er }}$ février 1690 : III, 825) 
8 En présentant ses inquiétudes comme motivées par la perfection de sa manière d'aimer, Mme de Sévigné ne manifeste pas seulement ce que Jacqueline Duchêne analyse comme le poids écrasant d'un amour maternel excessif et indiscret ${ }^{15}$ : elle fait preuve d'une compassion que requiert toute affection authentique - comme l'illustre l'un des «billets de protestation d'amitié » offert en modèle par Ortigue de Vaumorière :

Vôtre colique me fait crier miséricorde, je suis moins patient que vous dans des maux que je ne souffre que par communication. Ceux qui me touchent de plus prés, me sont moins sensibles, \& l'amitié me rend propre ce qui n'est que représentation \& que peinture à qui n'aime point. Je vous souhaite, mon cher Monsieur, une prompte \& entiere guérison, \& je suis plus à vous que vous ne sauriez vous l'imaginer ${ }^{16}$.

9 Le fait que Mme de Sévigné ne réserve pas ses mouvements de sympathie à sa fille montre bien qu'ils sont irréductibles à une forme de sentimentalité hors normes. C'est ainsi que face aux épreuves endurées par son cousin, la marquise ne manque pas d'assurer celui-ci d'une compassion présentée comme une preuve de son affection pour lui :

Vos malheurs me font une tristesse au cœur qui me fait bien sentir que je vous aime. (15 juillet $1673: \mathrm{I}, 586$, à Bussy-Rabutin)

10 À l'opposé d'une éruption passionnelle plus ou moins incongrue dans le cadre d'une relation entre une mère et sa fille, la compassion, explicitement revendiquée par l'épistolière comme une obligation morale pour le cœur aimant, nimbe ainsi les craintes maternelles d'un prestige axiologique aisément perceptible.

\subsection{Le rituel épistolaire de la condoléance}

11 Afin de mesurer pleinement les effets des assurances de sympathie maternelles dans les lettres à Mme de Grignan, il est indispensable de repérer les préconstruits sociodiscursifs qu'elles mobilisent - seule la connaissance des codes de la rhétorique épistolaire permettant d'éviter de surestimer l'originalité d'attitudes discursives et de procédés stylistiques qui se révèlent être des composantes aussi courantes que conventionnelles des correspondances familières. À y regarder de près, les formules de compassion de Mme de Sévigné s'avèrent profondément tributaires des normes d'expression propres à l'une des routines de la civilité épistolaire, le témoignage de condoléance. À l'époque de Mme de Sévigné, se condouloir, c'est-à-dire "s'afliger avec quelqu'un, faire compliment à quelqu'un sur sa douleur » et se conjouïr, c'est-à-dire « se réjouïr avec quelqu'un, de quelque bonheur qui lui est arrivé, féliciter quelqu'un de quelque bonheur qui lui est arrivé ${ }^{17}$ ", constituent des impératifs de la politesse. C'est ainsi que les manuels d'art épistolaire proposent systématiquement d'une part, des modèles de lettres pour les événements heureux (qu'elles soient appelées lettres de félicitation, de congratulation ou de conjouissance); et d'autre part, des modèles de lettres pour les aléas malheureux de l'existence, en l'occurrence, presque exclusivement des lettres de consolation ${ }^{18}$, réservées aux destinataires qui viennent de subir un deuil ${ }^{19}$, à de rares exceptions près - Furetière donnant un exemple de "Lettre de condoleance sur la perte d'un Procès ${ }^{20} »$ et Le Secrétaire de ce temps fournissant des modèles suffisamment généraux pour que les apprentis épistoliers puissent s'en inspirer quelle que soit l'épreuve traversée par le destinataire ${ }^{21}$. Adoptant la typologie des rituels sociaux mise au point par Erwing Goffmann, Maurice Daumas classe ces catégories de lettres dans les " rituels de ratification", qui sont " accomplis pour et envers un individu dont le statut s'est altéré d'une certaine façon », et qui « expriment que leur exécutant est sensible à 
la situation de celui qui a subi un tel changement, qu'il désire poursuivre la relation avec lui et lui conserver son appui $^{22} »$. La fréquentation de correspondances authentiques contemporaines de celle de Mme de Sévigné atteste d'ailleurs la vitalité usuelle de ce que l'on nomme alors des compliments de condoléance ${ }^{23}$, qui se présentent sous la forme de séquences textuelles autonomes au sein des lettres familières. Savoir que le fait de "participer à la douleur de quelqu'un » et de "témoigner qu'on prend part à son déplaisir ${ }^{24}$ » constitue une pratique de civilité courante permet d'éviter de mettre au seul compte de la soi-disant passion maternelle les manifestations de sympathie de Mme de Sévigné - ce qui ne remet nullement en cause leur authenticité vécue. De fait, les mouvements de compassion qui émaillent les lettres à Mme de Grignan relèvent sans doute autant d'affects intimes que d'habitudes socio-discursives, autant de mécanismes psychologiques que de rituels épistolaires. Alors qu'il est tentant d'interpréter les formules par lesquelles Mme de Sévigné « donn[e] témoignage de ce qu'[elle] prend part à la douleur ${ }^{25}$ » de sa fille comme des énoncés hyperboliques dénotant l'excès passionnel, force est de constater que l'épistolière fait finalement preuve d'une relative sobriété, tant les assurances de sympathie les plus enflammées semblent monnaie courante - comme le prouvent les modèles de «lettres à un amy malade » prodigués par le fameux Secrétaire à la mode de Puget de la Serre :

Les nouvelles de vostre maladie ont tellement alteré ma santé, que je puis me conter au nombre de ceux qui ne se portent pas bien. J'en suis veritablement si marry, que le regret qui m'en demeure me tient lieu de fievre. Prenez donc courage, si vous m'en voulez donner; vous sçavez l'interest que j'ay à tout ce qui vous touche; en un mot, je vous puis assurer, que si vous ne quittez bientost le lict, je seray contraint de le tenir.

Je ne vous diray pas jusques à quel point les tristes nouvelles de vostre maladie m'ont esté sensibles. Il me suffit de vous ramentevoir, qu'ayant l'honneur d'estre au nombre de vos meilleurs amis, le recit de votre mal ne m'a peu estre que fort contagieux, puis que j'en souffre une partie ${ }^{26}$.

Face à tant d'emphase, la prétendue hystérie maternelle de Mme de Sévigné se trouve fortement relativisée... Il ne s'agit d'ailleurs pas de juger de tels compliments de condoléance affectés ou insincères, mais seulement de souligner qu'à une époque où les rapports humains passent nécessairement par des comportements langagiers axiologiquement contraints, socialement formalisés et rhétoriquement codifiés, les sentiments ne sauraient être communiqués en-dehors de pratiques discursives stéréotypées.

\subsection{Deux topiques propres aux compliments de condoléance}

12 À cet égard, deux thèmes ne doivent pas être trop vite attribués à ce que d'aucuns analysent comme les "craintes imaginaires ${ }^{27}$ " d'une mère dotée d'un tempérament pathologiquement angoissé - deux thèmes dont il convient de mettre au jour les soubassements socio-discursifs afin d'en évaluer avec justesse les répercussions éthiques.

13 Le premier thème constitutif des compliments de condoléance à faire l'objet d'une appropriation dans les lettres à Mme de Grignan consiste en la revendication d'une douleur jugée supérieure à celle du destinataire ${ }^{28}$ :

Que je vous plains, ma fille, d'avoir à rebâtir votre château! Quelle dépense hors de saison! Il vous arrive des sortes de malheurs qui ne sont faits que pour vous; je les sens peut-être encore plus que vous ne les sentez. ( $1^{\mathrm{er}}$ octobre 1684 : III, 145) 
Ce topos de l'indépassable violence du " contrecoup »

Je vous vois partout dans un déchirement de cœur si terrible que j'en sens vivement le

contrecoup. (11 octobre 1688 : III, 365)

acquiert d'ailleurs une expressivité toute particulière à l'occasion des craintes qu'éprouve Mme de Grignan pour son fils, parti à la guerre - la compassion de Mme de Sévigné s'en trouvant littéralement redoublée :

Je ne comprends que trop toutes vos peines; elles retournent sur moi, de sorte que je les sens de deux côtés. (17 avril 1689 : III, 578)

Le deuxième thème propre aux compliments de condoléance à être réinvesti dans les assurances de sympathie maternelles consiste à affirmer qu'à force d'inquiétude pour les maux de l'autre, sa propre santé s'en trouve affectée - un lieu commun à laquelle la fragilité de Mme de Grignan et l'inquiétude maternelle, accrue par la distance, confèrent cependant une densité existentielle pathétique. C'est ainsi que lorsqu'elle affirme que la fièvre de son petit-fils lui ôte tout « repos ",

Je n'aurai aucun repos, ma bonne, que je ne sache la suite de cette fièvre. (26 juin $1675:$ I, 743)

Mme de Sévigné, aussi sincèrement affectée qu'elle soit par cette nouvelle, reproduit des formules courantes en contexte familier - ce que prouve le début de l'un des « billets de protestation d'amitié » d'Ortigue de Vaumorière :

Vôtre Lettre m'a fait souffrir vos peines, \& je ne puis être en repos depuis que je sai que vous êtes dans le trouble ${ }^{29}$.

De même, loin de ne devoir être considérés que comme des perturbations psychosomatiques originales trahissant des sentiments d'une intensité extraordinaire, les aveux de souffrance indissociablement morale et physique qu'engendre la compassion de Mme de Sévigné s'avèrent largement topiques. Un autre «billet de protestation d'amitié » offert en modèle par Ortigue de Vaumorière illustre à quel point ce thème semble banal dans un contexte de condoléance :

On m'a dit que vous ne vous ne vous conservez pas assez. Cependant, mon cher Monsieur, vous devez être persuadé qu'en l'état où je suis, ce sont les bonnes ou mauvaises nouvelles qui me viennent de vous, qui font mes bons ou mes mauvais jours. Redoublez vos soins pour la conservation d'une santé qui ne s'arreste pas en vôtre personne ; enfin, mon cher Monsieur, soïez heureux pour vous \& pour moi ${ }^{30}$.

En faisant dépendre son état de santé de celui de sa fille, Mme de Sévigné ne pratique pas un chantage typique d'une mère abusive, comme certains critiques ont pu le penser : elle parvient, en jouant sur la «compétence topique ${ }^{31}$ " de sa destinataire, à doter de valeur ses inquiétudes, qu'elle leste de la légitimité morale de la compassion. Persuader sa correspondante de la perfection d'une manière d'aimer capable de faire éprouver une authentique compassion, aussi incommode à vivre fût-elle : tel est l'enjeu des injonctions priant la comtesse de prendre soin d'elle, ne serait-ce que pour ménager sa mère :

Ayez un peu soin de ma vie, en ménageant la vôtre. (12 janvier 1680 : II, 792)

Hélas! quand ce ne serait que pour l'amour de moi, ma chère bonne, ayez soin de vous.

(13 mars 1680 : II, 872)

Les nombreuses supplications qui demandent à Mme de Grignan d'« avoir pitié » de sa mère ne manquent pas de réactiver le thème topique d'une compassion préjudiciable à son propre état de santé :

Ayez pitié de moi ; conservez-vous, si vous voulez que je vive. (13 février 1671 : I, 158)

Ma chère fille, ayez pitié de moi, si vous n'avez pitié de vous. (6 mars 1671 : I, 178)

[...] sérieusement, ayez pitié de vous, de votre santé, et de la mienne. (23 décembre

$1671:$ I, 398)

Ma très chère, ayez pitié de vous et de moi. (14 juillet 1677 : II, 485) 
Vous écrivez trop, ma chère bonne, vous savez le mal que cela vous fait; ayez pitié de moi et ne rallumez point cette poitrine. (21 mai 1680 : II, 938)

[...] conservez votre santé, ne vous fatiguez plus tant, ayez pitié de moi ; j'aurai bien de la peine à soutenir plus de tristesse que je n'en ai. (27 septembre 1684 : III, 143)

C'est une étrange amertume à digérer ici que la crainte de vous voir dangereusement malade. Il n'y a pas moyen de soutenir cette pensée jour et nuit. Ayez donc pitié de moi. (5 novembre 1684 : III, 154)

Ma chère Comtesse, si c'est chose possible, ayez pitié de vous et de nous [...].» (11 octobre 1688 : III, 365)

C'est dans cette perspective que doivent être comprises les confidences sur les troubles (crises d'angoisse, oppressions, insomnies) qui affectent Mme de Sévigné lorsqu'elle est inquiète pour sa fille :

Ne craignez-vous point cette douleur, cette chaleur, cette pesanteur intérieure? Je

vous assure que j'en suis bien malade. (13 mars 1680 : II, 871)

Loin de devoir être interprétés en termes de "lamentations » ou de " mises en garde » traduisant le désir d'emprise maternel ${ }^{32}$, de tels énoncés constituent surtout un indice des sédimentations psycho-linguistiques à l'œuvre dans l'expression de la compassion aussi singuliers que soient les investissements pragmatiques dont elle fait l'objet. Que le thème d'une santé compromise par les inquiétudes éprouvées pour une personne chère soit de nature topique, c'est ce que confirme cette phrase adressée à Bussy-Rabutin :

Ma fille a été bien malade ; elle est guérie, et moi aussi, car nous sentons, vous et moi,

tous les maux de nos filles. (23 décembre 1682 : III, 92)

15 Au terme de cette recontextualisation des compliments de condoléance, c'est-à-dire de l'élucidation des représentations collectives dans lesquelles elles s'enracinent, il apparaît donc que réduire les mouvements de compassion maternels à des symptômes affectifs, en conduisant à occulter l'intériorisation aussi bien des modèles axiologiques que des usages épistolaires qui les sous-tendent, aboutit à en mésestimer les enjeux proprement rhétoriques au sein de l'interaction épistolaire. Une fois neutralisée la tentation de la paraphrase psychologisante, il devient possible d'appréhender les formules de compassion des lettres à Mme de Grignan en tant que séquences discursives spécifiques, dont il s'agit à présent, afin d'en décrire précisément les modalités de fonctionnement, d'inventorier les principaux marqueurs stylistiques.

\section{Les marqueurs stylistiques des séquences de condoléance}

Caractérisée par les constantes interférences entre une situation existentielle (l'inquiétude d'une mère pour sa fille), un mécanisme psycho-affectif (la sympathie) et une situation de communication (l'entretien d'une correspondance familière), la condoléance apparaît comme une pratique discursive qui se traduit par des marqueurs stylistiques propres - marqueurs qui, s'ils ne sont pas nécessairement concomitants, s'avèrent sémiotiquement convergents. On peut ainsi distinguer quatre types de procédés qui déclenchent l'identification d'une séquence de condoléance, aussi réduite soit-elle - Mme de Sévigné veillant d'une part, à se soustraire au conformisme de formules aussi convenues qu'empesées et d'autre part, à abréger des assurances et des protestations qu'elle sait pénibles à sa fille. 


\subsection{Un marqueur sémantique : l'isotopie de la compassion}

17 Le premier outil langagier qui permet de repérer une séquence de condoléance est d'ordre sémantique. C'est l'isotopie de la compassion, qui se déploie essentiellement au moyen d'expressions verbales à forte teneur phraséologique ${ }^{33}$.

18 Certaines locutions verbales entraînent immédiatement le repérage d'un compliment de condoléance, parmi lesquelles prendre intérêt à (ici à l'occasion de la fièvre et de la saignée du petit Marquis, âgé de trois ans),

Je vous plains bien, et M. de Grignan; dites-lui l'intérêt tout particulier que je prends

à son inquiétude et à la vôtre. (26 juin $1675:$ I, 743)

entrer dans,

J'entre dans vos inquiétudes et je les sens. (13 avril 1689 : III, 575)

tenir au cœur,

Votre mal de gorge me tient au cœur. (28 janvier 1689 : III, 486)

être en peine de quelqu'un,

Je ne puis, ma bonne, que je ne sois en peine de vous, quand je songe au déplaisir que

vous aurez de la mort du pauvre Chevalier. (12 février 1672 : I, 437)

et, dans une moindre mesure, être en peine de quelque chose,

[...] il est impossible que vous dormiez avec tous ces dragons, et que votre sang ne

se mette en colère et ne fasse des ravages cruels. J'en suis tout à fait en peine [...]. (22

janvier 1690 : III, 819)

De même, certains verbes imposent la reconnaissance d'une séquence de condoléance, d'autant plus qu'ils sont généralement employés au sein de collocations, comme comprendre,

Tout cela le rendait aimable, et pour vous, et pour tout le monde; je comprends aisément votre douleur, puisque je la sens en moi. (12 février $1672:$ I, 437)

et surtout sentir :

Je voudrais que vous puissiez savoir combien je sens, quoiqu'à deux cents lieues de vous, toutes vos peines. (22 janvier 1690 : III, 819)

Désireuse de souligner à quel point les maux de sa fille lui sont sensibles en dépit de la distance, Mme de Sévigné insiste régulièrement sur sa capacité à partager aussi bien les souffrances que lui relate effectivement Mme de Grignan,

Je n'ai pu voir votre douleur sans renouveler la mienne. Je vous trouve véritablement affligée, et c'est avec tant de raison qu'il n'y a pas un mot à vous répondre. J'ai senti tout ce que vous sentez [...]. Je vous plains de l'avoir vu cet automne ; c'est une circonstance à votre douleur. (24 février $1672:$ I, 444)

que celles qu'elle suppose,

La maladie de Montgobert en est cause, je lui souhaite une bonne santé, et je sens le

chagrin que vous devez avoir de l'état où elle est. (II, 257)

ou qu'elle avait anticipées :

J'ai bien senti, ma chère fille, le chagrin et le dérangement que vous ferait la maladie du Chevalier [...]. (2 octobre $1680:$ III, 29)

Loin d'attester l'intensité exceptionnelle des sentiments maternels, ces formules sont tout à fait courantes lorsqu'il s'agit aussi bien de se condouloir que de se conjouir ${ }^{34}$. D'ailleurs, Mme de Sévigné, à l'instar de contemporains dotés d'une sensibilité aiguë aux phénomènes d'usure, ne peut que regretter la banalité des « "Je prends part...", "Je m'intéresse si fort..." et autres semblables choses trop vulgaires pour de beaux esprits ${ }^{35}$ ». Que l'épistolière déplore la déperdition sémantique d'expressions dont il est impossible de se passer, c'est ce qu'illustre ce passage, dans lequel elle exprime sa compassion à l'égard des soucis financiers de sa fille : 
Il est bien aisé de comprendre la tristesse de vos souffrances; rien n'est plus affligeant. Et pensez-vous que cela n'entre pas dans la composition de ce qui cause le douloureux état où vous êtes? Ma chère bonne, je vous supplie de croire que je le partage avec vous, et que je sens si vivement et si tendrement tout ce qui vous touche que ce n'est point y prendre part; ma bonne, c'est y entrer et le ressentir entièrement. (5 janvier 1680 : II, 785)

L'expolition, qui accumule les parasynonymes en une gradation croissante, ainsi que l'épanorthose finale (« ce n'est point... c'est ») visant à resémantiser la locution prendre part prouvent bien que la marquise, pour exprimer ses sentiments de sympathie, ne dispose d'aucun autre vocabulaire que celui de la civilité la plus stéréotypée.

\subsection{Un marqueur énonciatif : la modalité exclamative}

Le deuxième fait de langue à programmer l'identification d'une séquence de condoléance est d'ordre énonciatif, l'expression de la compassion passant régulièrement par la modalité exclamative, qui «exprim[e] une attitude affective du sujet parlant à l'égard de l'état de chose évoqué par son énoncé36 ». Tantôt une série d'exclamations précède ce qui peut être tenu pour le compliment de condoléance proprement dit, qu'ils préparent et justifient :

Je suis fâchée du voyage de M. de Grignan. [...] Quelle bombe tombée au milieu des plaisirs et de la tranquillité de votre automne! [...] Et quelle dépense! et qu'elle vient mal à propos! Je vois tous ces contretemps avec autant de chagrin que vous [...]. (2 octobre 1680 : III, 30)

Tantôt le compliment de condoléance prend lui-même la forme exclamative notamment lorsqu'il recourt au verbe plaindre:

Mais, ma chère bonne, qui vous aura sauvée de vos inquiétudes ? C'est où il n'y a point de remède, et c'est ce que vous n'avez point la force de supporter. Ce pauvre enfant! Mon Dieu, que je le plains, et que je vous plains! (15 novembre 1679 : II, 736)

On admettra qu'il est souvent difficile d'assigner aux exclamations concernant les dangers qui guettent Mme de Grignan une valeur pragmatique unique. Non seulement les énoncés exclamatifs peuvent cumuler plusieurs forces illocutoires, mais encore ils peuvent produire au sein de l'interaction épistolaire toutes sortes d'actes de langage indirects, qu'ils soient d'ordre expressif (avouer son inquiétude), directif (mettre en garde) ou assertif (témoigner sa compassion). C'est ce qu'illustrent les exclamations utilisées par Mme de Sévigné à propos des violents maux de tête de sa fille :

Mais, ma chère enfant, que je suis fâchée de votre mal de tête! Que pensez-vous me dire de ressembler à $M$. Pascal ? Vous me faites mourir. Il est vrai que c'est une belle chose que d'écrire comme lui ; rien n'est si divin. Mais la cruelle chose que d'avoir une tête aussi délicate et aussi épuisée que la sienne, qui a fait le tourment de sa vie et l'a coupée enfin au milieu de sa course! Il n'est pas toujours question des propositions d'Euclide pour se casser la tête; un certain point d'épuisement fait le même effet. Je crains aussi que l'air de Grignan ne vous gourmande et ne vous tourbillonne. Ah! que cela est fâcheux ! Je crains déjà que vous ne soyez emmaigrie et dévorée. (24 avril 1689 : III, 584)

Au risque toujours latent de « faire de longues plaintes, ou de grands éloges » ou encore d'« employer toute la Morale et toute l'éloquence inutilement ${ }^{37}$ ", les exclamatives constituent un efficace contrepoids. C'est ce que montrent les séquences de condoléance concernant l'un des beaux-frères de Mme de Grignan (et destinées à lui être communiquées), dans lesquelles les exclamations réaffirment la sympathie de 
l'épistolière de manière plus expressive que les formules conventionnelles («je suis touchée », « je suis sensible ») qu'elles jouxtent :

Je suis touchée des maux de ce pauvre Chevalier; voilà ce qui m'a fait regretter d'être partie. Quelle patience! Quel courage! Je suis très sensible à ses maux. (11 mai 1689 : III, 596)

Jamais une goutte n'a été si violente ni si cruelle. Quelle tristesse! N'a-t-il pas raison de regretter tout ce qu'il perd et ce qu'il fait perdre à sa famille? car il n'est pas inhumain. Quelle patience pour souffrir sans cesse des maux insupportables, que vous ne sauriez comparer qu'à ceux de l'enfer, mais qui sont regardés comme donnés par celui qui est maitre de toutes choses et à qui nous devons être soumis ! C'est une de mes tristes pensées que l'état de ce pauvre garçon. Qu'il a bien fait de choisir la demeure de Grignan pour être malade, plutôt que celle de Paris où l'on sent encore plus de n'être pas comme les autres, et où il n'aurait pas la consolation d'être avec sa famille et de vous avoir pour garde et pour médecin! c'est ce qui n'a point de prix. Je ne lui ferai point d'autre compliment que de lui dire que je suis sensible à ses peines. (4 janvier 1690 : III, 796)

Aussi sincèrement admiratives que soient ces exclamations, elles n'en sont pas moins fortement ritualisées, les propos louangeurs étant un passage obligé de la civilité en général et des compliments de condoléance ( je ne lui ferai point d'autre compliment que...») en particulier.

\subsection{Un marqueur lexico-syntaxique : les constructions à verbe support}

Le troisième moyen linguistique à signaler l'expression de la condoléance est d'ordre lexico-syntaxique. Il consiste en l'utilisation récurrente de verbes supports, c'est-à-dire de verbes «qui, à côté de leurs emplois ordinaires, se combinent avec un syntagme prédicatif [...] pour construire une forme complexe fonctionnellement équivalente à un verbe $^{38}$ ». Trois verbes en particulier sont analysables en termes d'«opérateurs causatifs ${ }^{39} »$ qui servent à construire des noms.

D’une part, Mme de Sévigné emploie le verbe donner, qui fait partie des verbes « au sens lexical peu marqué ${ }^{40}$ » qui peuvent donc être utilisés comme verbe support, tantôt au sein d'une proposition principale ou indépendante,

Votre voyage de Marseille me trouble; l'air de la petite vérole et le bruit des canons me donnent une inquiétude qui n'est que trop juste. (8 mai 1671 : I, 249)

Voici la saison des fièvres tierces; cette pensée me donne de méchantes heures et le souvenir de celles de l'année passée dont une sueur vous tira si à propos. On n'est pas toujours assuré d'un même secours. (21 mai 1680 : II, 938)

tantôt au sein d'une proposition subordonnées relative :

Je ne puis vous dissimuler tout à fait la légère inquiétude que me donne votre santé. (8 juin 1677 : II, 459)

Je tremble pour votre santé ; la bise me fait une oppression par la crainte qu'elle me donne. (29 septembre 1679 : II, 692)

Une heure, un jour, une semaine, un mois, un an, tout cela court et s'enfuit avec une rapidité qui m'afflige toujours, je dis même au travers des peines et des inquiétudes que me donne toujours votre mal. (19 janvier 1680 : II, 803)

Je souhaite la vôtre avec toute la passion qui est inséparable de la tendresse que j'ai pour vous, et de la tristesse que me donne l'idée de votre extrême délicatesse. (5 juin 1680 : II, 960) 
D'autre part, Mme de Sévigné utilise le "verbe à trois arguments » mettre ${ }^{41}$, qui s'avère d'autant plus approprié à la consignation des mouvements de compassion qu'il est doté d'un sémantisme transitionnel ${ }^{42}$ nettement perceptible :

Les pluies qu'il fait depuis trois jours me mettent au désespoir. (9 février $1671: \mathrm{I}, 152$ )

J'ai une extrême envie de savoir comme vous vous portez de cette frayeur.

C'est mon aversion que les frayeurs. [...] elles me mettent dans un état qui renverse entièrement ma santé. (16 septembre $1671:$ I, 346)

Enfin, le verbe faire, dont le complément nominal accède alors au statut de nom prédicatif ${ }^{43}$, figure souvent dans des locutions verbales courantes, telles que faire de la peine,

Vous allez être bien accablée d'écritures; cela me fait de la peine pour vous, car en vérité, cela tue. (27 août 1690 : III, 936)

Vos jambes froides et mortes, dont vous vous moquez, au moins devant moi, me font une peine incroyable. (11 octobre 1679 : II, 700)

faire peur,

Votre paresse me fait peur ; ne la croyez pas sur ce choix. (27 février 1671 : I, 172)

Ce médecin me fait peur. (13 septembre $1671:$ I, 345)

Mais vous êtes grosse jusqu'au menton; la mode de votre pays me fait peur. (18

octobre $1671:$ I, 364)

Votre soleil me fait peur. (13 avril 1672 : I, 477)

[...] votre sang me fait toujours peur. (29 septembre 1677 : II, 559)

L'air de Grignan me fait peur pour vous, ma fille. (19 avril 1689 : III, 580)

ou faire mal :

Vous êtes bien heureuse d'avoir de quoi payer tous vos arrérages de Paris, quand vous donnez du plus pur de votre sang, comme les seize cent francs ; cela me fait mal à penser! (6 octobre $1675:$ II, 119)

$\mathrm{Au}$ sein d'énoncés où le présent a une valeur de caractérisation générale, ce qui leur confère des allures de loi propre aux cœurs aimants, la locution faire mal sert à souligner la permanence ou du moins la fréquence et l'itération de la compassion maternelle :

Mon Dieu, que tout ce qui vous fatigue me fait de mal! (4 octobre 1679 : II, 692)

Profitant du fait que la lexicalisation de cette locution verbale n'est pas complète ${ }^{44}$, Mme de Sévigné y glisse tantôt des déterminants complexes («beaucoup de », «bien $\mathrm{du} »)$,

Tout ce qui vous est bon m'est excellent, et ce qui vous incommode me fait beaucoup

de mal. ( 7 juillet 1680 : II, 1003)

[...] et tout ce qui vous fatigue me fait bien du mal. (10 août 1680 : II, 1042)

Je pense fort aussi à votre santé, ma chère bonne, à votre tête, à votre air

impétueux qui vous mange. Cela me fait bien du mal. (27 avril 1689 : III, 586)

tantôt un adjectif qui entraîne la réapparition du déterminant,

Que tout ce qui vous est mauvais me fait un grand mal ! (14 février 1680 : II, 835)

qui ont en commun de véhiculer une charge sémantique d'intensité.

Si les locutions à verbe support s'avèrent particulièrement adaptées pour formuler la condoléance, c'est que toutes suggèrent une transformation de l'état psychologique liée à la compassion pour les maux de l'autre - qu'il s'agisse de crainte, d'inquiétude, de tristesse (avec le verbe donner), de désespoir (avec le verbe mettre), de peine, de peur ou de mal (avec le verbe faire). 


\subsection{Un marqueur morphosyntaxique : les périphrases verbales factitives} morphosyntaxique. Il s'agit des périphrases verbales de type faire + infinitif, qui réalisent une diathèse causative puisqu'elles impliquent un nouvel actant dans le procès engagé ${ }^{45}$. Dans la mesure où «du point de vue sémantique, la construction factitive véhicule un enchâssement sous faire d'une relation sémantique, où le nouvel actant, appelé aussi agent principal, causateur, [...] provoque ou déclenche la réalisation du procès exprimé par le verbe à l'infinitif ${ }^{46} "$, elle s'avère adéquate pour verbaliser les réactions de compassion. Indéniablement, la configuration actancielle instaurée par la périphrase factitive renforce la densité pathétique des séquences de condoléance, l'épistolière apparaissant en proie à d'incontrôlables inquiétudes: d'une part, «le causateur fait en sorte que l'actant destitué soit engagé ou impliqué dans le procès "; d'autre part, "cet actant destitué » apparaît comme un simple "patient », voire une « victime ${ }^{47} »$.

La cause des douloureux mouvements de sympathie maternels est toujours un inanimé. Afin de faire comprendre à sa destinataire ses angoisses, Mme de Sévigné explique d'abord la source de ses appréhensions (formulées puis reprises par des anaphores démonstratives telles que "cette pensée» ou "cela») avant d'en décrire les symptômes («transir depuis les pieds jusqu'à la tête ", « souffrir et le jour et la nuit », «trembler »):

Et d'un autre côté, vos Alpes, dont les chemins sont plus étroits que votre litière, où votre vie dépend de la fermeté du pied de votre cheval... Ma bonne, cette pensée me fait transir depuis les pieds jusqu'à la tête. (2 juin $1672: \mathrm{I}, 525)$

$\mathrm{Au}$ reste, ma chère enfant, quand je me représente votre maigreur et votre agitation, quand je pense combien vous êtes échauffée et que la moindre fièvre vous mettrait à l'extrémité, cela me fait souffrir et le jour et la nuit. (22 décembre 1673: I, 647)

Mais il faut vous bien porter et que cette frayeur ne vous ait rien gâté. Il me semble que vous êtes dans votre septième mois; cela me fait trembler, et d'autant plus que c'est un garçon. (13 septembre 1671 : I, 344)

En présentant les actions provoquées par le sujet ("cette pensée », «cela ») comme subies par le COD («me»), les périphrases factitives contribuent à donner de l'épistolière l'image d'un être vulnérable profondément touché par les maux de sa fille.

$\mathrm{Au}$ terme de cette étude des quatre marqueurs privilégiés des séquences de condoléance, on constate donc qu'aussi variés soient-ils, tous ont en commun de présenter le sujet comme étant affecté par une modification de son état émotionnel.

Pour conclure, il convient de souligner qu'appréhendée dans toute la complexité de ses présupposés axiologiques et de ses modalités stylistiques, l'expression de la compassion dans les lettres à Mme de Grignan s'avère constitutive du travail de figuration épistolaire. Conformes à la fois aux sentiments attendus de la part d'une mère aimante, aux valeurs véhiculées par l'idéal de la tendresse, aux comportements requis par une conception exigeante de l'amitié et aux usages prescrits par les règles de civilité épistolaire, les compliments de condoléance maternels prouvent que c'est au sein des rituels socio-discursifs préexistants que s'insinuent les mouvements de sensibilité maternels. Ainsi se trouve démontrée la nécessité, pour qui se propose d'évaluer la 
portée des faits de style orchestrés par Mme de Sévigné, de pratiquer une lecture centrée sur les procédures textuelles assurant la mise en discours de l'affectivité.

Comme le souligne Bernard Beugnot commentant les réflexions sur le style contenues dans les manuels épistolographiques de l'époque, les contemporains de Mme de Sévigné conçoivent explicitement la transcription épistolaire des mouvements de sensibilité comme un "système d'équivalences », certains qu'ils sont que «la vigueur et la sincérité du sentiment ne portent pas en elles l'aptitude à devenir texte parce qu'il y faut à la fois des moyens maîtrisés et une mise à distance ${ }^{48}$ ». Le fait que la transformation du "sentiment " en "forme " soit pensée en termes de "médiation stylistique » reposant sur " une culture moins abolie que dominée ${ }^{49}$ » ne manque donc pas d'accréditer un mode de lecture visant à élucider le fonctionnement des préconstruits socio-discursifs engagés dans la communication épistolaire de l'affectivité - puisque «dans le passage de l'émotion au discours, du secret de la conscience à l'exhibition de la parole une rhétorique se glisse $\mathrm{e}^{50} »$.

\section{NOTES}

1. Données entre parenthèses, les références aux lettres de Mme de Sévigné mentionnent la date de la lettre et sa pagination (tome et page) dans l'édition de référence : Correspondance, éd. R. Duchêne, Paris, Gallimard, coll. «Bibliothèque de la Pléiade », 1972-1978, 3 tomes. Dans toutes les citations, les italiques sont nôtres.

2. Voir l'enquête lexicologique esquissée par C. Cartmill, «Mme de Sévigné et la théorie de la sympathie ", dans R. Duchêne dir., Mme de Sévigné (1626-1696). Provence, spectacles, "lanternes", Colloque international du Tricentenaire de la mort de Mme de Sévigné, Château de Grignan (29 mai-1 ${ }^{\text {er }}$ juin 1996), Grignan, Association d'Action Culturelle des Châteaux Départementaux de la Drôme, 1998, p. 277-286.

3. A. de Lamartine, Euvres complètes, publiées et inédites. Vies de quelques hommes illustres, t. III (Milton, Mme de Sévigné, Bossuet, Fénelon, Nelson), Paris, chez l'auteur, 1863, p. 85 : «Par un phénomène d'instinct maternel, qui ressemble presque autant à un miracle de la nature qu'à un prodige d'affection, bien que cette mère eût enfanté cette fille depuis quinze ans, elle semblait porter toujours ce fruit mal détaché de ses entrailles dans ses flancs. [...] Il faut admettre cette espèce de folie de l'instinct maternel dans l'âme de Mme de Sévigné pour comprendre cette connexité absolue d'existence et cet anéantissement complet de sa personnalité dans une autre. » 4. M. Longino, "Mme de Sévigné, the art of vicarious living ", Stanford French and Italian Studies, 58, "Women in French literature. A collection of essays", M. Guggenheim dir., 1988, p. 65-75.

5. P. Avrane, « Un canapé pour la marquise ? », Europe, janvier-février 1996, p. 7.

6. M.-P. Pasquini-Butts, Mme de Sévigné, Michel Tournier et la tentation cannibale: les figures parentales de l'incorporation filiale, Ph. D., Yale University, 1997 [Dissertation Abstracts International, A 58/05, novembre 1997, p. 1740].

7. N. Freidel, La Conquête de l'intime. Public et privé dans la Correspondance de Mme de Sévigné, Paris, Champion, coll. « Lumière classique », 2009, p. 630-632.

8. Appréhendé à travers son étymologie latine, qui reste prégnante au XVII ${ }^{\mathrm{e}}$ siècle, le terme désigne d'abord, au sens propre, le partage, la mise en commun. Au sens figuré, si le mot communication appartient, à l'époque de Mme de Sévigné, au paradigme de la conversation, dans 
la mesure où il comporte, à des degrés variables selon les définitions, le sème /parole/, il met l'accent moins sur l'activité langagière que sur ses conséquences intersubjectives, pensées en termes d'intelligence et de familiarité. C'est ce qu'illustrent les définitions données par les dictionnaires de l'époque. Pour Richelet, communication signifie «Entretien. Familiarité. Commerce. [Avoir une communication étroite avec quelqu'un] (Dictionnaire françois, contenant les mots et les choses..., Genève, Jean Herman Widerhold, 1680). Pour Furetière, il « se dit aussi de la fréquentation, de l'intelligence qu'on a avec quelqu'un" (Dictionnaire universel..., La HayeRotterdam, Arnout et Reiner Leers, 1690). Selon l'Académie, communication »signifie aussi, Commerce, familiarité, et correspondance. Ils ont grande communication ensemble. Ils ont, ou ils entretiennent communication par lettres " (Dictionnaire de l'Académie françoise, Paris, Veuve JeanBaptiste Coignard, 1694).

9. Tel est l'exemple que donne Furetière du sens figuré du verbe communiquer : «Donner quelque chose à un autre, le faire participer de ce qu'on possede » (Dictionnaire universel..., op. cit.).

10. Voir Fr. de Sales, Introduction à la vie dévote, $3^{\mathrm{e}}$ partie, chap. XXII, «Quelques autres avis sur les amitiés », Paris, Gallimard, « Bibliothèque de la Pléiade ", éd. A. Ravier et R. Devoas, 1969, p. 192 passage mentionné par N. Freidel, op. cit., p. 626 : «L'amitié requiert une grande communication entre les amants, autrement elle ne peut ni naître ni subsister. C'est pourquoi il arrive souvent qu'avec la communication de l'amitié, plusieurs autres communications passent et se glissent insensiblement de cœur en cœur par une mutuelle infusion et réciproque écoulement d'affections, d'inclinations et d'impressions. »

11. L. Silvestre de Sacy, Traité de l'amitié, Paris, Jean Moreau, 1703, p. 72-73 : « Le premier fruit que les amis doivent tirer de la communication de leurs pensées, c'est une vive attention sur tout ce qui les interesse. Comme ils connoissent parfaitement la situation de leur esprit \& de leur fortune, rien ne peut échapper à cette attention. Elle ne s'arrête pas seulement aux occasions importantes où il s'agit de grands services, ce n'est pas encore ici le lieu d'en parler, elle s'étend aux moins considerables : \& c'est là particulierement où le charme de la tendresse se fait sentir. Dans les grandes occasions la gloire \& la generosité ont leur part aux offices de l'amitié : dans les petites, l'amitié brille seule \& en a tout l'honneur. Je ne regarde donc point comme austeres seulement, mais encore comme chagrins, ces gens qui traitent les petits soins de bagatelles, \& les renvoyent aux amans \& aux femmes. On doit mépriser les foiblesses de l'amour, mais on peut en imiter la vivacité. Contribuer aux innocents plaisirs d'un ami, essayer de le divertir dans ses plus legeres peines, aller au devant de ce qu'il desire, quoi que peu important ; être inquiet de ses moindres maux, sensible à ses moindres plaisirs ; c'est sçavoir repandre les douceurs de l'amitié sur toutes les parties de la vie. »

12. Madeleine de Scudéry, Clélie. Histoire romaine [1654-1660], éd. D. Denis, Paris, Gallimard, coll. «Folio classique », 2006, p. 72 et 74.

13. C'est ce que D. Maingueneau désigne comme le processus d'incorporation, «Lecture, incorporation et monde éthique ", Études de linguistique appliquée, $\mathrm{n}^{\circ} 119$, «Les textes et leurs lectures ", Fr. Cicurel dir., juillet-septembre 2000, p. 268.

14. Mme de Sévigné n'hésite pas à comparer ses sentiments à ceux de son gendre, coupable à ses yeux de légèreté voire d'inconséquence en ce qui concerne la santé de la comtesse (5 janvier 1680 : II, 784-785) : «Que j'ai d'envie, ma bonne, que cette bise et ce vent du midi vous laissent en repos! Mais quel malheur d'être blessée de deux vents qui sont si souvent dans le monde, et surtout en Provence ! En vérité, ma bonne, vous devez un peu m'excuser si je pense tristement à l'état où vous êtes; ceux qui sont tranquilles en vous voyant ainsi sont bien heureux.» Sur les «dissonances » relationnelles induites par la mise en concurrence de la «tendresse maternelle » et du « lien conjugal », voir R. Duchêne, Mme de Sévigné et la lettre d'amour, Paris, Klincksieck, coll. «Bibliothèque de l'âge classique », p. 169-176.

15. J. Duchêne, Françoise de Grignan ou le mal d'amour, Paris, Fayard, 1985. 
16. P. Ortigue de Vaumorière, Lettres sur toutes sortes de sujets, avec des avis sur La maniére de les écrire, tome I, Paris, Jean Guignard, 1690, p. 196-197.

17. P. Richelet, Dictionnaire françois, contenant les mots et les choses..., op. cit., s. v. se condouloir et se conjouïr.

18. Commentant l'évolution des manuels épistolographiques parus entre 1560 et 1828, M. Daumas remarque que » la consolation, qui peut concerner la captivité, l'emprisonnement, la maladie, est centrée de plus en plus sur le décès » (dans « Manuels épistolaires et identité sociale (XVI ${ }^{\mathrm{e}}-\mathrm{XVIII}{ }^{\mathrm{e}}$ siècles) ", RHMC, tome 40-4, octobre-décembre 1993, p. 539).

19. Furetière y insiste à plusieurs reprises (Essais de lettres familières Sur toutes sortes de sujets. Avec un Discours sur l'Art Epistolaire [1690], Genève, Slatkine Reprints, 1972, p. 50-51 et p. 220) : « L'Auteur met une distinction entre Consolation \& Condoleance. Condoleance sur la perte d'un procès, \& consolation sur la mort d'un père, d'une femme, d'un ami. » " On ne doit dire Lettre de Consolation que quand il s'agit de la mort.» Dans la pratique, on observe de nombreuses confusions entre les deux termes... y compris de la part de Furetière lui-même (ibid., p. 139-141).

20. Ibid., p. 50-52.

21. Ce manuel d'art épistolaire, qui présente la particularité d'être bilingue (français/allemand), encadre les lettres de consolation provenant du manuel de Puget de la Serre de deux modèles qui restent vagues sur la nature des maux endurés par le correspondant (Le Secrétaire de ce tems..., Francfort sur le Mein, Jean Maximilian à Sande, 1709, p. 314 et p. 354) : la première est intitulée «Consolation sur quoi que ce soit »; la seconde » Lettres pour marquer la part qu'on prend à son déplaisir». Par ailleurs, ce manuel reproduit également la «lettre sur la condoléance d'un procès » de Furetière.

22. M. Daumas, op. cit., p. 543.

23. Le dictionnaire de l'Académie mentionne à l'article Condoléance : « Il n'a guère d'usage qu'en ces phrases, Compliment de condoléance, Lettre de condoléance, c'est-à-dire, Compliment qui se fait, lettre qui s'écrit pour témoigner la part qu'on prend à la douleur de quelqu'un » (Dictionnaire de l'Académie françoise, op. cit., s. v. condoléance). Le dictionnaire de Richelet indique qu' « On dit faire des compliments de condoléance, c'est à dire faire un compliment à quelqu'un sur sa douleur, lui témoigner qu'on la partage » (op. cit., s. v. condoléance).

24. Telle est la définition que donne le dictionnaire de l'Académie du verbe Condouloir, Se condouloir (Dictionnaire de l'Académie françoise, op. cit., s. v. condouloir).

25. Nous citons la définition que donne Furetière du verbe se condouloir (Dictionnaire universel..., op. cit., s. v. se condouloir).

26. J. Puget de la Serre, Le Secrétaire à la mode..., Amsterdam, 1646, p. 71-72. Cette lettre constitue un modèle suffisamment fonctionnel pour que Th. La Grue le fasse figurer dans son manuel bilingue français/flamand, La Vraye Introduction à la Langue françoise : avec Quatre Dialogues François et Flamans, Amsterdam, Samuel Imbrechts, 1669, p. 250.

27. J. Duchêne, Françoise de Grignan ou le mal d'amour, op. cit., p. 51.

28. On trouve un écho de ce topos dans la séquence conclusive de la « Lettre de condoleance sur la perte d'un Procès » donnée en exemple par Furetière (Essais de lettres familières Sur toutes sortes de sujets. Avec un Discours sur l'Art Epistolaire, op. cit., p. 52, nous soulignons) : «Depuis que vous m'avez écrit cette nouvelle, il me semble que je vous en aime davantage, \& qu'un si triste succés a augmenté la passion que j'ai depuis longtemps pour mon cher Ami. Au moins est-il certain que je prendrai desormais plus de soin de la lui temoigner : Et dans ma douleur, que je croi plus grande que la vôtre, je chercherai ma consolation à vous faire connoître, que quand j'ai souhaité vôtre amitié, je n'avois en veuë que vôtre merite. Adieu. »

29. P. Ortigue de Vaumorière, Lettres sur toutes sortes de sujets, avec des avis sur La maniére de les écrire, t. I, Paris, Jean Guignard, 1690, p. 210.

30. Ibid., p. 196. 
31. G.-E. Sarfati, "Aspects épistémologiques et conceptuels d'une théorie linguistique de la doxa ", dans R. Koren et R. Amossy dir., Après Perelman. Quelles politiques pour les nouvelles rhétoriques? L'argumentation dans les sciences du langage, Paris, L'Harmattan, 2002, p. 76.

32. On aura reconnu la thèse défendue par J. Duchêne, op. cit., p. 49.

33. Rappelons que «la phraséologie est constituée de combinaisons récurrentes, plus ou moins stabilisées, de formes lexicales et grammaticales ; les unités phraséologiques apparaissent comme des figements, c'est-à-dire des ensembles plus ou moins longs de formes simples construites dans des contextes contraints, susceptibles néanmoins de certaines variations. » (P. Fiala, «Pour une approche discursive de la phraséologie. Remarques en vrac sur la locutionnalité et quelques points de vue qui s'y rapportent, sans doute », Langage et Société, $n^{\circ} 42$, décembre 1987, p. 32.)

34. Voir J. Puget de la Serre, Le Secrétaire à la mode..., op. cit., p. 27 et p. 30 : la locution prendre part s'emploie aussi bien dans les « lettres de congratulation » ("Seulement est à obser ver, qu'il faut d'entrée tesmoigner le contentement qu'on reçoit du bon-heur de son ami ; disant que nous y prenons une aussi grande part, que s'il nous estoit advenu à nous mesmes ») que dans les " lettres de consolation" («Mais si quelque grand mal est arrivé à nostre ami, il faut dire qu'on a esté touché au vif de son affliction ; \& qu'y prenans telle part comme nous faisons, nous ne sommes pas si propres à l'en consoler qu'à nous condouloir ensemble »).

35. C'est à propos des lettres écrites pour «se réjouir avec les gens à qui il est arrivé quelque bonheur" qu'Amilcar se moque de ces formules usuelles. Voir «De la manière d'écrire des lettres » [1684], dans M. de Scudéry, "De l'air galant» et autres Conversations. Pour une étude de l'archive galante, D. Denis éd., Paris, Champion, coll. « Sources classiques », 1998, p. 150.

36. M. Riegel, J.-Chr. Pellat et R. Rioul, Grammaire méthodique du français, Paris, PUF, 2009 [4 $4^{\text {ème }}$ éd.], p. 663.

37. Tels sont les écueils de la lettre de consolation que dénonce Plotine (« De la manière d'écrire des lettres » op. cit., p. 150).

38. M. Riegel, J.-Chr. Pellat et R. Rioul, Grammaire méthodique du français, op. cit., p. 415. Pour une mise au point théorique sur la notion de verbe support, voir M. Gross, «La fonction sémantique des verbes supports ", Travaux de Linguistique, $\mathrm{n}^{\circ} 37$, «Le lexique-grammaire », B. Lamiroy dir., 1998, p. 25-46. Pour une réflexion sur le fonctionnement de certains groupes prédicatifs dans la langue du XVII ${ }^{\mathrm{e}}$ siècle, voir Fr. Lévrier, "Les constructions à verbe support dans la langue classique : quelques particularités », dans J. Choi-Jonin, M. Bras, A. Dagnac et M. Rouquier dir., Questions de classification en linguistique: méthodes et descriptions. Mélanges offerts au professeur Christian Molinier, Peter Lang, coll. « Sciences pour la communication », 2005, p. 211-226.

39. M. Gross, "Les bases empiriques de la notion de prédicat sémantique ", Langages, $\mathrm{n}^{\circ} 63$, "Formes syntaxiques et prédicats sémantiques », A. Guillot et Chr. Leclère dir., septembre 1981, p. 23.

40. J. Gardes-Tamine, La Grammaire, t. I, Paris, Colin, 1988, p. 115.

41. Sur l'inclusion du verbe mettre dans la catégorie des causatifs, voir M. Gross, "Les bases empiriques de la notion de prédicat sémantique ", op. cit., p. 22-25.

42. Voir R. Martin, "Temporalité et "Classes de verbes" ", L'Information grammaticale, $\mathrm{n}^{\circ} 39$, octobre 1988, p. 5 : «Un procès est transitionnel (ou "transformatif" ou encore "résultatif") s'il marque le passage d'un état à un état résultant (irréversible ou non : pendant combien de temps du lait stérilisé reste-t-il stérile?). C'est sans doute Gustave Guillaume qui a le mieux décrit ce phénomène, en faisant voir que certains procès emportent avec eux une subséquence (une "séquelle"), que leur sémantisme n'est autre que la transition d'un état d'avant à un état d'après. [...] Un verbe est transitionnel s'il prédique le passage d'un état à un autre. »

43. Voir J. Giry-Schneider, «Les noms construits avec faire : compléments ou prédicats? », Langue française, $n^{\circ} 69$, février 1986, p. 49: par «noms prédicatifs", on désigne «des noms, généralement traités de manière uniforme comme simples compléments de verbes, [qui] accèdent au rang de "vrais" prédicats sémantiques, tandis que le verbe n'est qu'un outil 
morphologique et syntaxique qui sert à les construire, c'est-à-dire à leur donner la forme d'une phrase. » De fait, pour reprendre une autre analyse de J. Giry-Schneider (« La notion de modifieur obligatoire dans des phrases à verbe support avoir complexes ", Langages, n 121, mars 1996, p. 19), les « phrases à verbe support présentent un décalage entre la forme et le sens : ce n'est pas le verbe qui sélectionne les arguments, mais le nom formellement "complément" de ce verbe ». 44. Sur les propriétés propres au mécanisme linguistique du figement (polylexicalité, opacité sémantique, blocage des propriétés transformationnelles, non-actualisation des éléments, blocage des paradigmes synonymiques, non-insertion), voir M. Gross, Les Expressions figées en français. Noms composés et autres locutions, Gap-Paris, Ophrys, 1996, chap. 1: «La notion de figement ", en particulier p. 13-15 sur la notion de locution.

45. Voir M. Gross, «Les bases empiriques de la notion de prédicat sémantique », op. cit., p. 23-24: «En français, le verbe faire s'applique à de nombreux verbes pour former une phrase à sens causatif. [...]. La caractéristique de faire est qu'il constitue un élément verbal avec l'infinitif qu'il accompagne », ce qui produit un « changement du nombre d'arguments. »

46. I. Novakova, «Le factitif français : approche syntaxique, sémantique et contrastive (françaisbulgare) ", Revue Tranel (Travaux neuchâtelois de linguistique), n³7, 2002, p. 95.

47. Id.

48. B. Beugnot, «Style ou styles épistolaires? " [1978], repris dans La Mémoire du texte. Essais de poétique classique, Paris, Champion, 1994, p. 193.

49. Ibid., p. 194 : «La médiation stylistique représente donc, dans le geste épistolaire, une prise de conscience, selon le postulat classique que l'idée ou le sentiment ne préexistent jamais totalement à l'avènement de leur forme [...]. »

50. Id.

\section{AUTEUR}

\section{CÉCILE LIGNEREUX}

Université Grenoble Alpes - UMR Litt\&Arts / RARE Rhétorique de l'Antiquité à la Révolution 